\title{
Palmitic acid decreases cell migration by increasing RGS2 expression and decreasing SERCA expression
}

\author{
Octavio Galindo-Hernandez ${ }^{1}$, Ana Gabriela Leija-Montoya ${ }^{1}$, Tatiana Romero-Garcia ${ }^{2}$ \\ and Jose Gustavo Vazquez-Jimenez ${ }^{3}$ (1) \\ ${ }^{1}$ Autonomous University of Baja California, Laboratory of Biochemistry, School of Medicine, \\ Campus Mexicali, BC, Mexico. \\ ${ }_{2}^{2}$ Autonomous University of Baja California, Laboratory of Biochemistry, Sports School, \\ Campus Mexicali, BC, Mexico. \\ ${ }^{3}$ Autonomous University of Baja California, Laboratory of Molecular Pathogenesis, School of Medicine, \\ Campus Mexicali, BC, Mexico.
}

\begin{abstract}
Palmitic acid, the main saturated fatty acid, is related with a wide range of metabolic disorders such as obesity, type 2 diabetes and heart disease. It is known that palmitic acid disturbs the expression of some important proteins for cell homeostasis such as SERCA and RGS2, however, the role of this lipid at the molecular level in these disorders is not completely elucidated. Thus, our aim was to determinate the effect of palmitic acid in a relevant cell process as it is cell migration and the participation of SERCA and RGS2 in this response. We found that palmitic acid reduces cell migration (determined by the Boyden chamber method) in an epithelial cell line (HEK293) and this effect is modulated by SERCA and RGS2 differential protein expression (measured by western blot). Also, overexpression of individual proteins, RGS2 and SERCA, produced a decrease and an increase on cell migration, respectively. Taken together, these data suggest that the expression of regulatory proteins is affected by high concentrations of saturated fatty acids and in consequence cell migration is diminished in epithelial cells.
\end{abstract}

Keywords: Palmitic acid, cell migration, RGS2, SERCA.

Received: August 06, 2020; Accepted: January 18, 2021.

Obesity is a state that gives rise to the development of many metabolic conditions; such as hypertension, heart disease and type 2 diabetes mellitus (de Luca and Olefsky 2008; Ryu et al., 2019), however, the molecular alterations that precede these pathologies are not completely understood. Recently, it has been shown that a high-fat diet induces an increase in the expression of the Regulator of G protein signaling 2 (RGS2) (Nunn et al., 2011). RGS-proteins are GTPases accelerating proteins for heterotrimeric $\alpha$-subunits (Soundararajan et al., 2008). RGS2 selectively inhibits signaling mediated by heterotrimeric $\mathrm{G}_{\mathrm{q} / 11}$ proteins (Heximer et al., 1997).

There is a correlation between high concentrations of fatty acids (FA) and the elevation of RGS2 protein levels since the expression of this protein was increased in highfat fed mice. Likewise, an RGS2 knock-out mouse model $\left(r g s 2^{-/-}\right)$showed a reduction in serum lipids and increased insulin sensitivity (Nunn et al., 2011). Therefore, changes in RGS2 expression correlate with the pathogenesis of metabolic disorders (Osei-Owusu et al., 2007).

In this same context, obesity prompts augmented plasma levels of non-esterified FA, particularly palmitic acid (PA), the main saturated FA. Recent studies have shown

Send correspondence to Jose Gustavo Vazquez-Jimenez. Autonomous University of Baja California, Laboratory of Molecular Pathogenesis, School of Medicine, Campus Mexicali, Av. Humberto Torres Sangines S/N, Col. Centro Civico, 21000, Mexicali, BC, Mexico. E-mail: gustavo. vazquez@uabc.edu.mx. that increased PA levels result in a reduced expression of the sarco/endoplasmic reticulum calcium ATPase (SERCA) and hence a decrease in its activity (Li et al., 2004; Fu et al., 2012; Mayer and Belsham 2010; Vazquez et al., 2016). Among other conditions, the endoplasmic reticulum (ER) requires a high luminal $\mathrm{Ca}^{2+}$ concentration to guarantee the correct synthesis, folding and assembly of membrane and secretory proteins (Ceylan-Isik et al., 2011). In this respect, SERCA protein plays a central role, since it is in charge of $\mathrm{Ca}^{2+}$ transport into the ER. Disturbances in ER homeostasis leads to stress and therefore activation of the unfolded protein response (UPR) (Özcan et al., 2004; 2006; Park et al., 2010), which directly induces ER stress, through a mechanism that has been proposed to be associated with the downregulation of the insulin signaling pathway (Vazquez et al., 2016).

Given that, we have focused on the study of RGS2 and SERCA proteins, considering that high concentrations of saturated fatty acids have recently been reported to be involved in the regulation of the expression of these proteins (Nunn et al., 2011; Vazquez et al., 2016). Nevertheless, there are no studies that integrate these two alterations and that attribute any immediate functional effect to them. In order to solve this, we used an epithelial cell line and PA as a study model; since the epithelia are in constant migration and regeneration, and these are the first to suffer alterations induced by PA (Duan et al., 2017; Ghezzal et al., 2020). Specifically, we have chosen embryonic kidney cells 293 (HEK293), because it is a cell 
line that possesses morphological and functional properties of epithelial cells (Braun and Huber, 2002; Stepanenko and Dmitrenko 2015) and also due to their efficiency to evaluate the expression of RGS2 and SERCA (Jang et al., 2014; Bovo et al., 2020). Furthermore, this cell line is convenient both for carrying out transfection and for evaluating cell migration (Xue et al., 2019; Wang et al., 2020).

Palmitic acid ( $\geq 99 \%$ pure), bovine serum albuminfatty acid-free (FAF-BSA), Dulbecco's modified Eagle's medium (DMEM; Sigma Aldrich); fetal bovine serum (FBS; ByProductos); Lipofectamine 2000 (Life Technologies); full-length human SERCA2b cDNA clone (ID 5503508) in pCMV-SPORT6 vector (Invitrogen); full-length human RGS2 3xHA-tagged cDNA (CloneID RGS020TN00) in the pcDNA3.1+ vector (cDNA Resource Center); anti-SERCA2 (Thermo Scientific), anti-RGS2, anti-Actin and mitomycin C (Santa Cruz Biotechnology).

HEK293 cells (ATCC) were grown in P-100 dishes at $37{ }^{\circ} \mathrm{C}$ in a humidified atmosphere $\left(95 \%\right.$ air, $\left.5 \% \mathrm{CO}_{2}\right)$ in DMEM 10\% FBS supplemented, $100 \mu \mathrm{g} / \mathrm{ml}$ streptomycin and 100 units $/ \mathrm{ml}$ penicillin. For experiments, cells were sub-cultured in 6 plates to $80 \%$ confluence and cultured with DMEM (serum free) for $6 \mathrm{~h}$ and then PA treatments were performed.

HEK293 cells were seeded $\left(2.5 \times 10^{4}\right)$ for 3 days. Then, cells were transfected with RGS2 3xHA tagged or pEF1/His-AhSERCA2b cDNA ( $1 \mu \mathrm{g} /$ well) in $5 \mu \mathrm{g} / \mathrm{ml}$ of Lipofectamine 2000 in complete culture medium at $37^{\circ} \mathrm{C}$ for $6 \mathrm{~h}$. This procedure was followed by $18 \mathrm{~h}$ culturing in fresh medium before lipid incubation.

PA stock solutions at $500 \mathrm{mM}$ were prepared in DMSO. HEK 293 cells were swapped to serum-free DMEM with $1 \%$ FAF-BSA and then treated with $0.25 \mathrm{mM}$ of PA for $8 \mathrm{~h}$. Then the cells were placed on ice, washed twice with ice-cold PBS and lysed with $100 \mu 1$ of $1 x$ Laemmli sample buffer.

Cell lysates were defrosted, sonicated, then incubated at $99^{\circ} \mathrm{C}$ for $5 \mathrm{~min}$, and finally centrifuged at $14,000 \mathrm{rpm}$ for $5 \mathrm{~min}$. The obtained supernatant was loaded on SDS-PAGE gels, electrophoresed and then transferred to PVDF membranes using a semi-dry chamber. Membranes were incubated with the corresponding primary antibodies overnight at $4{ }^{\circ} \mathrm{C}$ and washed 3 times with TBS-T buffer before incubating with secondary antibodies (HRP-conjugated) for $1 \mathrm{~h}$ at room temperature. Chemiluminescent signals were visualized using an HRP chemiluminescent western blot detection (Millipore). Densitometric signals of immunoblot films were determined with ImageJ Software (1.53e version).

Migration assays were performed by the Boyden chamber method in 24-well plates containing 12 cell culture inserts (Corning Inc., Cat. 3422). After starvation, HEK293 cultures were treated for $2 \mathrm{~h}$ with $12 \mu \mathrm{M}$ mitomycin $\mathrm{C}$ to inhibit proliferation. Briefly, HEK293 cells $\left(1 \times 10^{5}\right)$ were placed on the top chamber $(100 \mu \mathrm{l} /$ insert $)$, whereas the lower chamber contained $600 \mu$ DMEM supplemented or not with $10 \%$ FBS. Cells were incubated for $24 \mathrm{~h}$ at $37{ }^{\circ} \mathrm{C}$ with $5 \%$ $\mathrm{CO}_{2}$; and then cells on the upper surface of membrane were discarded, while cells on the lower surface of membrane were washed and fixed with cold methanol. The cells were stained with $0.1 \%$ crystal violet in $1 \mathrm{X}$ PBS, and the dye was eluted with $10 \%$ acetic acid. Finally, the solution was analyzed by a spectrophotometer at $600 \mathrm{~nm}$. Background value was determined from wells with no attached cells.

PRISM 6.0 software was used to analyze the average absorbances as well as the densitometric intensities from western blot films. Statistical significance was determined by one-way ANOVA with Dunnett's post-test. Significance was defined at $p$ value $<0.05$. Data were normalized based on the control and the mean \pm S.E.M.

In this research we have explored two previously reported molecular events: First, the association between obesity and increased expression of RGS2 (Imagawa et al., 1999; Nishizuka et al., 2001; Nunn et al., 2011). Second, the association among high concentrations of PA and decreased SERCA2 expression (Vazquez et al., 2016).

We wanted to resolve whether these two molecular alterations were present in HEK293 cell line and in that case if they are associated with disturbances on cell migration. Thus, we performed incubations of HEK293 cells with $0.25 \mathrm{mM}$ of PA for $8 \mathrm{~h}$. Previously, this concentration was reported to cause functional modifications and protein expression alterations; however, this effect did not occur with high concentrations of unsaturated fatty acid (Vazquez et al., 2016). Our results showed that incubations with PA decrease HEK293 cells migration (Figure 1A). Also, as shown in Figure 1B, PA increased RGS2 expression $\sim 3$-fold over control; in the same experiment, the fatty acid decreased SERCA2 expression $\sim 0.7-$ fold with respect to control (Figure 1C). These data suggest that PA decreases cell migration while increasing RGS2 and decreasing SERCA expression in HEK293 cells.

Recent research has shown that PA induces a decrease in SERCA protein levels; as well as PA produced a sustained inhibition of SERCA pump ATPase activity (Vazquez et al., 2016).

On the other hand, it has been suggested that RGS2 may play an essential role in the regulation of body metabolism, as $\mathrm{rgs}^{-/-}$mice metabolic activity was increased (Nunn et al., 2011).

To resolve whether alterations in the expression of SERCA and RGS2 were mechanisms associated with alterations in cell migration, transfection of both proteins in HEK293 cells was carried out. As shown in Figure 2A, transfection was efficient, nonetheless, the SERCA intensity signal was so strong that the control signal was only observed after overexposure of the plate. As shown in Figure 2B, in presence of $10 \%$ FBS, SERCA overexpression increases cell migration $\sim 1$-fold over control. On the other hand, overexpression of RGS2 decreased cell migration ( $\sim 0.5-$ fold with respect to the control) (Figure 2B). Importantly, in $10 \%$ FBS conditions, overexpression of RGS2 decreases migration by $\sim 1.5$-fold respecting the SERCA overexpression condition (Figure $2 \mathrm{~B}$ ). It should be noted that these changes in migration were not present when overexpression of the empty vector was performed (MOCK) (Figure 2B). These data suggest that increased SERCA expression enhances cell migration, while RGS2 increased expression diminish it.

Previous research has reported that SERCA overexpression prevents PA-induced insulin resistance (Park et al., 2010; Vazquez et al., 2016) and since PA decreases cell migration, we wanted to resolve whether SERCA overexpression prevents PA-induced decreased cell migration. Hence, we performed 
A)

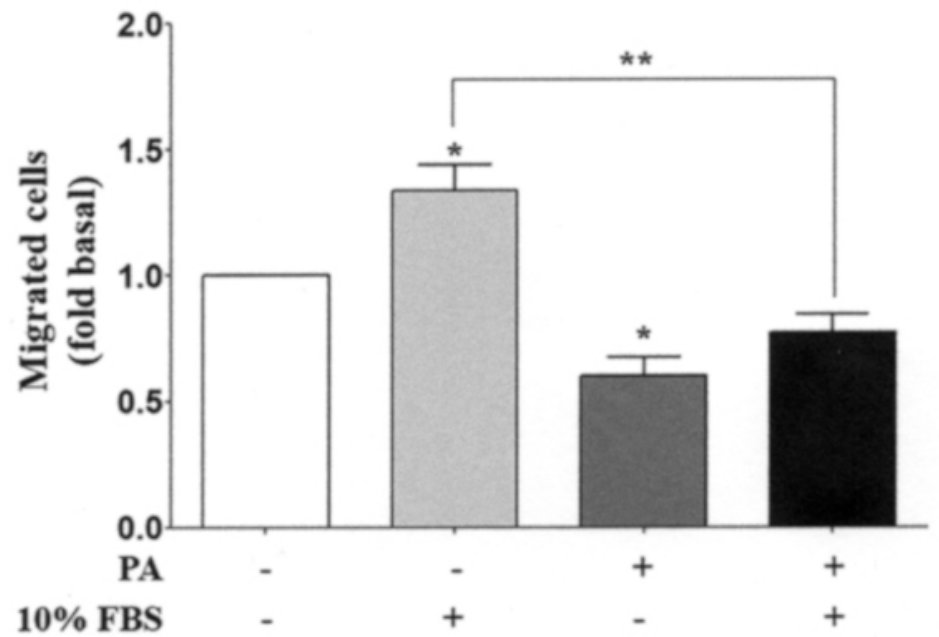

B)

C)
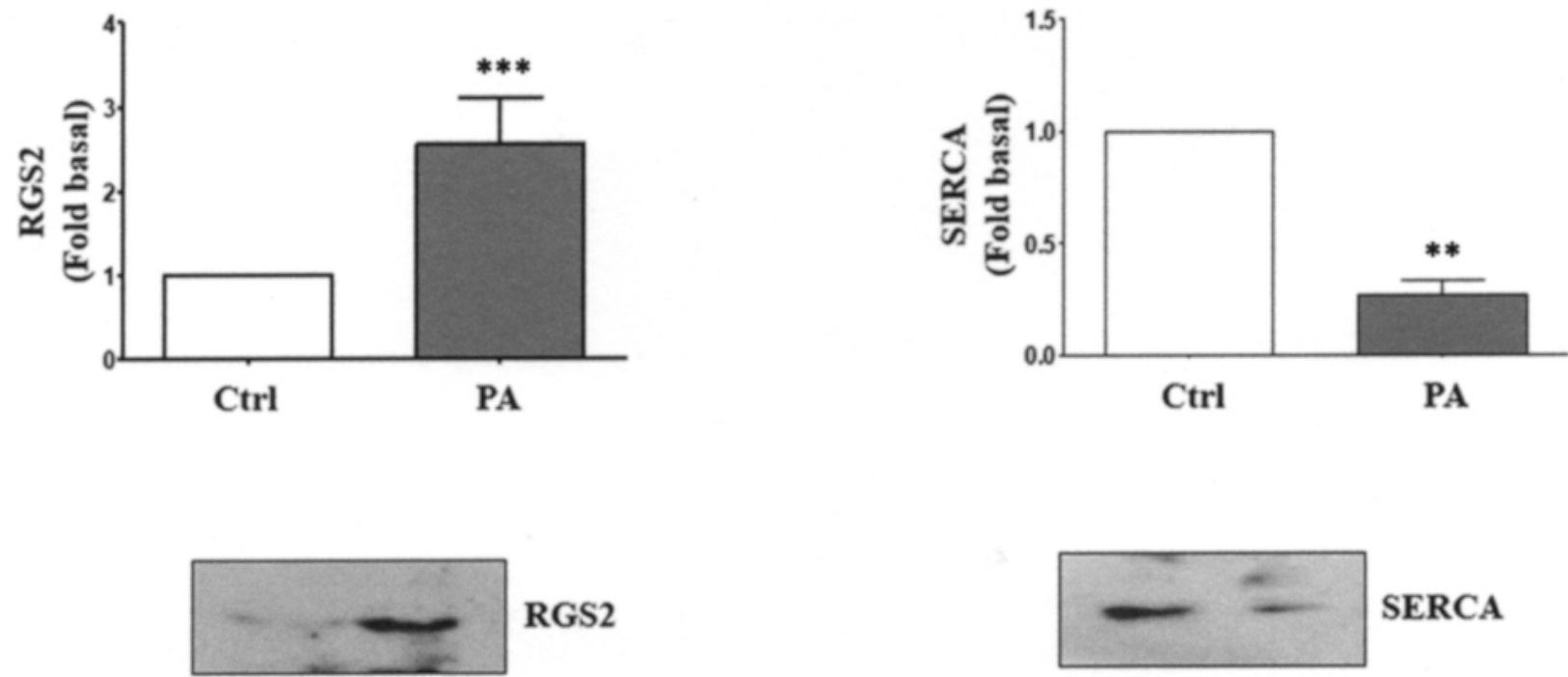

RGS2

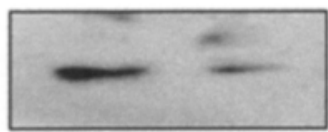

SERCA

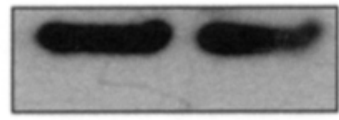

Actin

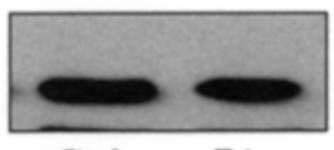

Actin

Ctrl PA

Ctrl PA

Figure 1 - Palmitic acid inhibits cell migration in HEK293 cells. a. After starvation, HEK293 cells were washed, equilibrated in DMEM without FBS, and pretreated for $2 \mathrm{~h}$ with $12 \mu \mathrm{M}$ mitomycin C. Cells were untreated or treated with $0.25 \mathrm{mM} \mathrm{PA}$ (an inducer of cell migration) or $10 \%$ FBS and cell migration assays were performed by using the Boyden chamber method. The graphs represent the mean \pm S.E.M. of migration of three independent experiments and are expressed as the fold of unstimulated cells. b, c After starvation, HEK293 cells were washed and equilibrated in DMEM without FBS. Cells were treated with $0.25 \mathrm{mM}$ PA in DMEM and lysates were obtained. RGS2 and SERCA were analyzed by western blotting with anti-RGS2 and anti-SERCA2, respectively. Western blots were also probed for actin as a loading control. Asterisks denote comparisons made to unstimulated cells. $* p<0.05, * * p<0.01, * * * p<0.001$ vs control condition (unstimulated cells).

SERCA overexpression prior to incubation with PA. As shown in Figure 2C, SERCA overexpression prevented the decrease in cell migration induced by PA.
In conclusion, high concentrations of saturated fatty acids alter the expression of regulatory proteins, thus decreasing cell migration in epithelial cells. 
A)

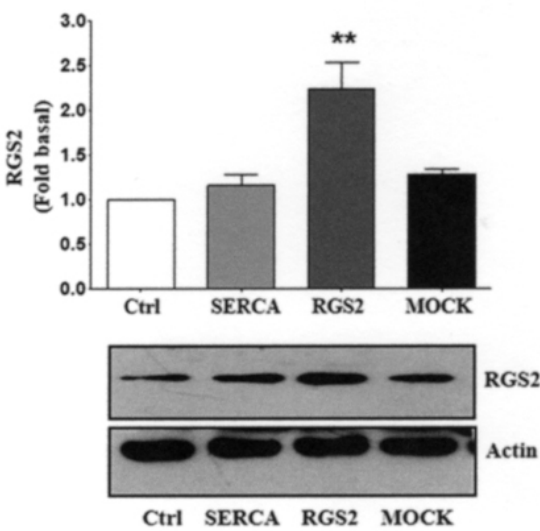

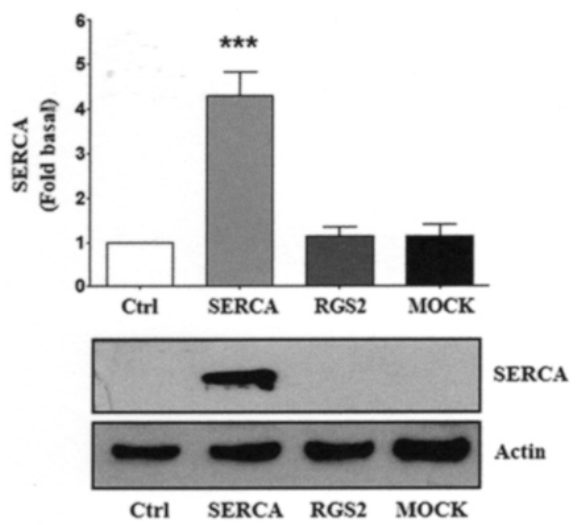

Ctrl SERCA RGS2 MOCK

B)

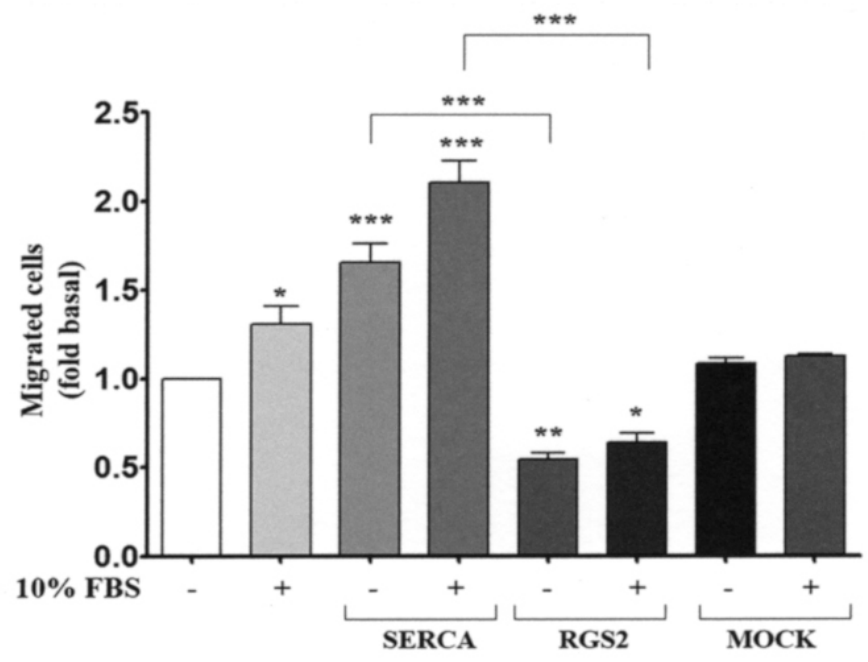

C)

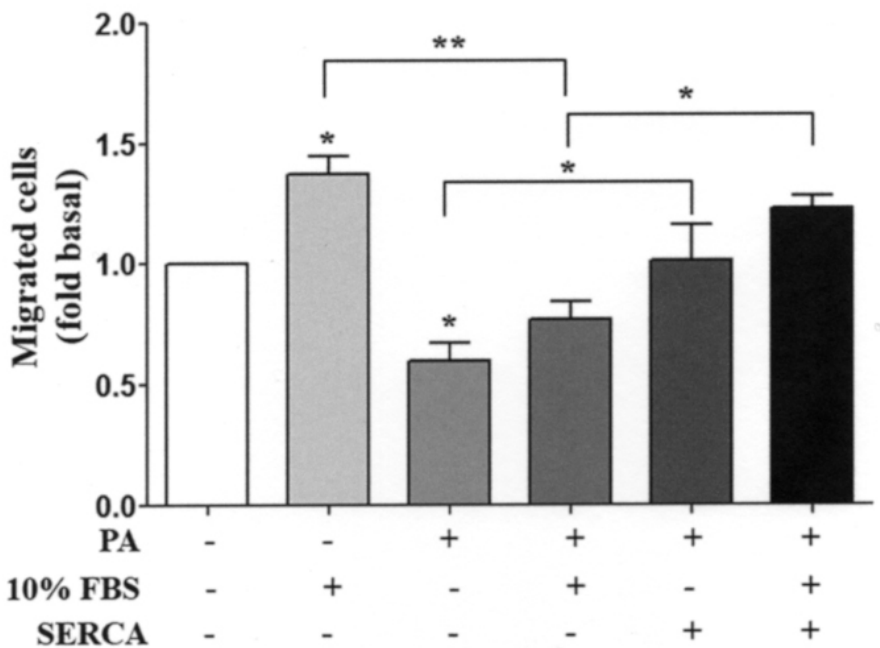

Figure 2 - SERCA overexpression prevented the decrease in PA-induced cell migration in HEK293 cells. HEK293 cells were transfected with a RGS2 and SERCA2 construct or with an empty vector (MOCK). a Cells were lysed and RGS2 and SERCA expression were analyzed by western blotting using anti-RGS2 and anti-SERCA2 Ab, respectively. Western blots were also probed for actin as a loading control. b HEK293 cells were untreated or treated with $10 \%$ FBS (as a positive control of cell migration) and cell migration assays were performed by using the Boyden chamber method. The graphs represent the mean \pm S.E.M. of migration of three independent experiments and are expressed as the fold of unstimulated cells. $\mathbf{c}$ HEK293 cells were transfected with a SERCA2 construct prior to incubation for $8 \mathrm{~h}$ with $0.25 \mathrm{mM}$ PA. Cell migration assays were performed by using the Boyden chamber method. The graphs represent the mean \pm S.E.M. of migration of three independent experiments and are expressed as the fold of control. $* p<0.05$, $* * p<0.01, * * * p<0.001$ vs control condition (unstimulated cells). 


\section{Acknowledgments}

This research was supported by the Universidad Autonoma de Baja California and partially by PRODEP, Apoyo a Fortalecimiento de Cuerpos Academicos 28911 (to J.G.V.J. and A.G.L.M.) and CONACYT 300015 (to O.G.H.).

\section{Conflict of Interest} to declare.

None of the authors has any potential conflict of interest

\section{Author Contributions}

OGH and JGVJ conceived the study; OGH, AGLM, TRG and JGVJ conducted the experiments; OGH and JGVJ analyzed the data and wrote the manuscript. All authors read and approved the final version.

\section{References}

Bovo E, Nikolaienko R, Cleary S, Seflova J, Kahn D, Robia S, and Zima A (2020). Dimerization of SERCA2a enhances transport rate and improves energetic efficiency in living cells. Biophys J 119:1456-1465.

Braun GS and Huber SM (2002). Development of renal function. Zoology 105:341-354.

Ceylan-Isik AF, Sreejayan N and Ren J (2011). Endoplasmic reticulum chaperon tauroursodeoxycholic acid alleviates obesity-induced myocardial contractile dysfunction. J Mol Cell Cardiol 50:107-116.

de Luca C and Olefsky J M (2008). Inflammation and insulin resistance. FEBS Lett 582:97-105.

Duan NN, Liu XJ and Wu J (2017). Palmitic acid elicits hepatic stellate cell activation through inflammasomes and hedgehog signaling. Life Sci 176:42-52.

Fu S, Watkins SM and Hotamisligil GS (2012). The role of endoplasmic reticulum in hepatic lipid homeostasis and stress signaling. Cell Metab 15:623-634.

Ghezzal S, Postal BG, Quevrain E, Brot L, Seksik P, Leturque A and Carriere V (2020). Palmitic acid damages gut epithelium integrity and initiates inflammatory cytokine production. Biochim Biophys Acta Mol Cell Biol Lipids 1865:158530.

Heximer SP, Watson N, Linder ME, Blumer K J and Hepler JR (1997). RGS2/G0S8 is a selective inhibitor of Gqalpha function. Proc Natl Acad Sci U S A 94:14389-14393.

Imagawa M, Tsuchiya T and Nishihara T (1999). Identification of inducible genes at the early stage of adipocyte differentiation of 3T3-L1 cells. Biochem Biophys Res Commun 254:299-305.

Jang H, Kim J, Noh M, Rhee M and Park K (2014). Regulator of G protein signaling 2 (RGS2) deficiency accelerates the progression of kidney fibrosis. Biochim Biophys Acta 1842:1733-1741.

Li Y, Ge M, Ciani L, Kuriakose G, Westover EJ, Dura M and Tabas I (2004). Enrichment of endoplasmic reticulum with cholesterol inhibits sarcoplasmic-endoplasmic reticulum calcium ATPase$2 \mathrm{~b}$ activity in parallel with increased order of membrane lipids: implications for depletion of endoplasmic reticulum calcium stores and apoptosis. J Biol Chem 279:37030-37039.

Mayer CM and Belsham DD (2010). Palmitate attenuates insulin signaling and induces endoplasmic reticulum stress and apoptosis in hypothalamic neurons: rescue of resistance and apoptosis through adenosine 5' monophosphate-activated protein kinase activation. Endocrinology 151:576-585.
Nishizuka M, Honda K, Tsuchiya T, Nishihara T and Imagawa M (2001). RGS2 promotes adipocyte differentiation in the presence of ligand for peroxisome proliferator- activated receptor gamma. J Biol Chem 276:29625-29627.

Nunn C, Zhao P, Zou MX Summers K, Guglielmo CG and Chidiac $P$ (2011). Resistance to age-related, normal body weight gain in RGS2 deficient mice. Cell Signal 23:1375-1386.

Osei-Owusu P, Sun X, Drenan RM, Steinberg TH and Blumer KJ (2007). Regulation of RGS2 and second messenger signaling in vascular smooth muscle cells by cGMP- dependent protein kinase. J Biol Chem 282:31656-31665.

Özcan U, Cao Q, Yilmaz E, Lee AH, Iwakoshi NN, Ozdelen E and Hotamisligil GS (2004). Endoplasmic reticulum stress links obesity, insulin action, and type 2 diabetes. Science 306:457-461.

Özcan U, Yilmaz E, Özcan L, Furuhashi M, Vaillancourt E, Smith RO and Hotamisligil, GS (2006). Chemical chaperones reduce ER stress and restore glucose homeostasis in a mouse model of type 2 diabetes. Science 313:1137-40

Park SW, Zhou Y, Lee J, Lee J and Ozcan U. (2010). Sarco(endo) plasmic reticulum $\mathrm{Ca} 2+-$ ATPase $2 \mathrm{~b}$ is a major regulator of endoplasmic reticulum stress and glucose homeostasis in obesity. Proc Natl Acad Sci U S A 107:19320-19325.

Ryu S, Frith E, Pedisic Z, Kang M and Loprinzi P (2019). Secular trends in the association between obesity and hypertension among adults in the United States, 1999-2014. Eur J Intern Med 62:37-42.

Soundararajan M, Willard FS, Kimple AJ, Turnbull AP, Ball LJ, Schoch GA, Gileadi C, Fedorov OY, Dowler EF et al. (2008). Structural diversity in the RGS domain and its interaction with heterotrimeric $\mathrm{G}$ protein alpha-subunits. Proc Natl Acad Sci U S A 105:6457-6462.

Stepanenko AA and Dmitrenko VV (2015). HEK293 in cell biology and cancer research: Phenotype, karyotype, tumorigenicity, and stress-induced genome-phenotype evolution. Gene 2015;569:182-190.

Vazquez-Jimenez JG, Chavez-Reyes J, Romero-Garcia T, ZarainHerzberg A, Valdes-Flores J, Galindo-Rosales M, Rueda A, Guerrero-Hernandez A and Olivares-Reyes JA(2016). Palmitic acid but not palmitoleic acid induces insulin resistance in a human endothelial cell line by decreasing SERCA pump expression. Cell Signal 28:53-59.

Wang H, Zhang T, Ge X, Chen J, Zhao Y and Fu J (2020). Parkin overexpression attenuates $A \beta$-induced mitochondrial dysfunction in HEK293 cells by restoring impaired mitophagy. Life Sci 224:1173.

Xue S, Xu H, Sun Z, Shen H, Chen S, Ouyang J, Zhou Q, Hu X and Cui $H$ (2019). Depletion of TRDMT1 affects 5 -methylcytosine modification of mRNA and inhibits HEK 293 cell proliferation and migration. Biochem Biophys Res Commun 520:60-66.

Associate Editor: Daisy Maria Fávero Salvadori

License information: This is an open-access article distributed under the terms of the Creative Commons Attribution License (type CC-BY), which permits unrestricted use distribution and reproduction in any medium, provided the original article is properly cited. 